\title{
Analytic Renormalisation of the Exponential Interaction and Weak Interaction Singularities
}

\author{
P. K. MitTer \\ Department of Theoretical Physics, Oxford, England
}

Received October 13, 1970

\begin{abstract}
The parity non conserving interaction of a neutral vector meson with fermions is considered as a mathematical model suitable for investigating divergence problems of the weak interactions. Through the Stückelberg formalism and a canonical transformation the interaction is converted into an exponential form. The exponential interaction is studied, in the second order of perturbation theory, through the method of analytic renormalisation. Generalised amplitudes are introduced as localizable distributions depending on auxiliary complex parameters $\lambda$. It is shown that the distributions possess a nonisolated singularity at the physical point $\lambda_{0}$. A method is developed for discarding the singularity thereby obtaining the physical amplitudes as localizable distributions which display a non-analytic dependence on the coupling constant.
\end{abstract}

\section{Introduction}

In this paper we study the problem of regularising the exponential interaction of scalar fields in perturbation theory by taking up the analytic renormalisation approach of Speer [1] in a wider distribution theoretic framework. In doing so one meets new technical problems characteristic of a local unrenormalisable theory. We shall study these problems in the second order of perturbation theory (in the exponential interaction). We will show that the earlier results of Volkov [2] and Okubo [3] arise naturally ${ }^{1}$ in this framework.

The problem of regularising the exponential interaction is not devoid of physical interest. Our specific interest stems from the earlier work of Lee [6], who showed, by exploiting the Stuckelberg formalism and performing a canonical transformation, that the study of the short distance singularities of the (unrenormalisable) intermediate boson model of weak interaction could be reduced to a study of the iterations of the exponential interaction in perturbation theory. Lee attempted to sum the leading and next to leading singularities of the individual Feynman

${ }^{1}$ Alternative derivations for the propagator (see text) as a distribution have been obtained, recently and independently, by the authors of Ref. $[4,5]$.

19 Commun. math Phys., Vol. 20 
graphs which were then successively removed by counter terms. We shall adopt a different and rigorous approach. This is possible because of two facts. Firstly, it is known [7] that there is an intrinsic ambiguity in the definition of formal perturbation theory $T$-products which is not resolved by unitarity and locality. However, this ambiguity may be exploited to obtain regularised $T$-products as distributions (generalised functions) and the finite renormalisation arbitrariness incorporated in the form of distributions of point support which have an interpretation as counter terms in the Lagrangian [7]. Secondly, from the work of Jaffe [8], it is known that the exponential interaction is strictly localizable and thus the regularisation problem is well posed in an appropriate generalised function framework.

In order to keep the physical motivation in mind we review in Section II Lee's formulation of the theory of neutral vector mesons with a parity non-conserving interaction with massive fermions. By employing a generalised Stückelberg formalism in which the masses of the vector and scalar mesons are not constrained to be equal and performing a canonical transformation one is led to the study of exponential interactions of massless scalar particles. The regularisation problem is technically simpler for the massless case. In Section III we give a definition of the formal two point $T$-products (super propagator) $\mathscr{D}^{ \pm}(x)$ as a generalised function. For this purpose we utilise as our fundamental test function space the Gelfand-Shilov space [9] $S^{\beta}$ (denoted as $\mathscr{C}$ in the following) with an appropriate restriction of $\beta$ for strict localizability. Equivalently one may use Jaffe's space $\mathscr{C}$. The spectral function is shown to be a generalised function in $\tilde{\mathscr{C}}^{\prime}$, where $\tilde{\mathscr{C}}$ is the space obtained on Fourier transformation. We then introduce a generalised function $\mathscr{D}^{ \pm}(\lambda ; x)$ with an appropriate restriction on the complex parameter $\lambda$. The Fourier transform $\tilde{\mathscr{D}}^{ \pm}(\lambda, p)$ is shown to possess a non-isolated singularity in $\lambda$ in the neighbourhood of the physical point $\lambda=0$. A method is given to discard the singularity and a definition is obtained of $\tilde{\mathscr{D}}^{ \pm}(p)$ at the physical point $\lambda=0$ as a generalised function in $\tilde{\mathscr{C}}^{\prime}$. The configuration space super propagator $\mathscr{D}^{ \pm}(x)$ is defined in $\mathscr{C}^{\prime}$ through Fourier transformation.

The final result, which displays a logarithmic dependence on the coupling constant, agrees with the earlier result of Okubo [3] and Volkov [2] which is seen to have a precise mathematical significance in local field theory. In Section IV we present the extension of this procedure to renormalisation of loop diagrams. We treat a simple case in detail, leaving the discussion of the general case to a future paper. In Section V we comment on the finite renormalisation ambiguity, its possible elimination by the Lehmann-Pohlmeyer minimum singularity hypothesis [4] and on further problems concerning analytic renormalisation in higher orders. 


\section{Section II. The Model Lagrangian}

We consider the parity nonconserving interaction of neutral vector mesons of mass $M_{W}$ with fermions of mass $m$.

where

$$
\mathscr{L}_{\text {int }}=g j_{\mu} W_{\mu}
$$

$$
j_{\mu}=\bar{N} \mathrm{i} \gamma_{\mu}\left(1+\gamma_{5}\right) N
$$

and $N$, and $W_{\mu}$ represent the fermion and spin 1 neutral vector boson fields. The degrees of infinity in perturbation theory, as revealed by powercounting, are the same as those of a more realistic model of the weak interactions with charged currents [6]. In order to facilitate the analysis of the ultraviolet divergences in this model Lee [6] introduced the Stückelberg formalism for the vector field. Lee also developed (see Appendix of Ref. [6]) a generalised Stückelberg formalism in which the mass $M_{W}$ of the vector particle is not constrained to be equal to the mass $\mu$ of the auxiliary scalar particle. As we will see this general formalism offers a number of technical advantages if we evaluate graphs in the limit $\mu \rightarrow 0$. This limit is to be taken on the Feynman graphs.

As usual we make the decomposition:

$$
W_{\mu}=\phi_{\mu}+\frac{1}{M_{W}} \partial_{\mu} \theta .
$$

The total Lagrangian is:

$$
\mathscr{L}=\mathscr{L}_{0}+\mathscr{L}_{\text {int }}
$$

where $\mathscr{L}_{\text {int }}$ is given by Eq. (1), and

$$
\begin{aligned}
\mathscr{L}_{0}=-\bar{N}\left(\gamma_{\mu} \hat{\partial}_{\mu}+m\right) N- & \frac{1}{4}\left(\partial_{\mu} \phi_{v}-\partial_{\nu} \phi_{\mu}\right)^{2}-\frac{1}{2} \xi\left(\partial_{\nu} \phi_{v}\right)^{2}-\frac{1}{2} m_{W}^{2} \phi_{v}^{2} \\
& -\frac{1}{2}\left(\partial_{v} \theta\right)^{2}-\frac{1}{2} \mu^{2} \theta^{2}
\end{aligned}
$$

where

$$
\xi=\left(\frac{M_{W}}{\mu}\right)^{2}
$$

$\phi_{\mu}$ and $\theta$ are spin one and zero fields with masses $M_{W}$ and $\mu$ respectively.

From the equations of motion for the $\phi_{\mu}$ and $\theta$ fields it follows that

$$
\left(\square-\mu^{2}\right)\left(\partial_{\lambda} \phi_{\lambda}+\frac{\mu^{2}}{M_{W}} \theta\right)=0 \text {. }
$$

Eq. (7) is always true. Thus, as noted by Lee [6], all radiation processes involving the spin 0 part of $\phi_{\mu}$ are compensated by processes involving the $\theta$ fields. The rule for a unitary $S$-matrix is to restrict ourselves to a sector in which there are no $\theta$ external lines and no spin 0 part in the $\phi_{\mu}$ external lines. 
In perturbation theory we will need the covariant free propagators for the $\varphi_{\mu}$ and $\theta$ fields.

The free field commutator functions (Pauli-Jordan functions) are:

and

$$
\left[\theta(x), \theta\left(x^{\prime}\right)\right]=i \Delta\left(x-x^{\prime} ; \mu\right)
$$

$\left[\phi_{\mu}(x), \phi_{v}\left(x^{\prime}\right)\right]=i\left(\delta_{\mu v} \Delta\left(x-x^{\prime} ; M_{W}\right)-\frac{\partial_{\mu} \partial_{v}}{M_{W}^{2}}\left(\Delta\left(x-x^{\prime} ; M_{W}\right)-\Delta\left(x-x^{\prime} ; \mu\right)\right)\right)(9)$

where $\Delta\left(x-x^{\prime} ; \mu\right)$ is the Pauli-Jordan function for the scalar field with mass $\mu$. In the limit $\mu=M_{w}$ we recover the conventional expressions of the usual Stückelberg formalism. It is easy to check that (8) and (9) are consistent with the equations of motion. Furthermore,

$$
\left[\left(\frac{\partial}{\partial x_{\mu}} \phi_{\mu}(x)+\frac{\mu^{2}}{M_{w}} \theta(x)\right), \quad\left(\frac{\partial}{\partial x_{\mu}^{\prime}} \phi_{v}\left(x^{\prime}\right)+\frac{\mu^{2}}{M_{w}} \theta\left(x^{\prime}\right)\right)\right]=0
$$

which implies consistency with the subsidiary condition. In the $\mu \rightarrow 0$ limit, in which we will compute Feynman graphs, the covariant free field propagators in momentum space are:

$$
\begin{aligned}
\overrightarrow{\theta \theta} & \rightarrow \frac{i}{p^{2}-i o}, \\
\widetilde{\varphi_{\mu}} \varphi_{v} & \rightarrow \frac{i}{p^{2}+M_{w}^{2}-i o}\left(\delta_{\mu v}-\frac{p_{\mu} p_{v}}{p^{2}}\right) .
\end{aligned}
$$

Eq. (12) is reminiscent of the "Landau gauge" in contrast to the usual Stückelberg vector propagator which is in the "Feynman gauge".

We now make the unitary transformation

$$
\varphi(x)=\exp \left[\frac{-i g}{M_{w}}\left(1+\gamma_{5}\right) \theta\right] N(x) .
$$

Substituting in the Lagrangian (4) we obtain, in the new basis,

$$
\begin{aligned}
\mathscr{L}= & -\frac{1}{4}\left(\partial_{\mu} \phi_{v}-\partial_{\nu} \phi_{\mu}\right)^{2}-\frac{1}{2} \xi\left(\partial_{\nu} \phi_{v}\right)^{2}-\frac{1}{2} m_{N}^{2} \phi_{v}^{2}-\frac{1}{2}\left(\partial_{v} \theta\right)^{2}-\frac{1}{2} \mu^{2} \theta^{2} \\
& -\bar{\psi} \gamma_{\mu} \partial_{\mu} \psi+g j_{\mu} \phi_{\mu}-m \bar{\psi} \exp \left[\frac{2 i g}{M_{w}} \gamma_{5} \theta\right] \psi
\end{aligned}
$$

where

$$
j_{\mu}=\bar{\psi} i \gamma_{\mu}\left(1+\gamma_{5}\right) \psi
$$

Separating out the fermion mass term, the interaction Lagrangian to be used in perturbation theory is:

$$
\mathscr{L}_{\text {int }}=g j_{\mu} \varphi_{\mu}-m \bar{\psi}\left(\exp \left[\frac{2 i g}{m_{w}} \gamma_{5} \theta\right]-1\right) \psi
$$


The Lagrangians (1) and (15) will lead to the same on mass shell matrix elements since the two formulations are related by a canonical transformation. In any given order of perturbation theory the most divergent piece due to the $p_{\mu} p_{v}$ term in the $W$ propagator can be traced to the exponential interaction in (15). The most divergent piece in any given process can be traced to $\theta$ graphs with all $\theta$ lines emitted and absorbed at the same point, and it can be removed from all processes by a single mass renormalisation [6]. Alternatively, in terms of the interaction Lagrangian (15), these may be removed by Wick ordering the Lagrangian (15). We shall therefore take as our interaction Lagrangian

$$
\mathscr{L}_{\text {int }}=g: j_{\mu}: \varphi_{\mu}-m: \bar{\psi}\left[:\left(\exp \left(\frac{2 i g}{M_{w}} \gamma_{5} \theta\right)-1\right):\right] \psi: .
$$

Introducing the notations:

$$
\Lambda_{ \pm}=\frac{1 \pm \gamma_{5}}{2}
$$

and

where

$$
\Theta^{ \pm}=:\left(\exp \left( \pm i x^{\frac{1}{2}} \theta\right)-1\right):
$$

$$
x^{\frac{1}{2}}=\frac{2 g}{m_{w}},
$$

(16) is conveniently written in the form

$$
\mathscr{L}_{\text {int }}=g: j_{\mu} \phi_{\mu}:-m\left(: \bar{\psi} \Lambda_{+} \psi: \Theta^{+}+: \bar{\psi} \Lambda_{-} \psi: \Theta^{-}\right)
$$

We will iterate $\mathscr{L}_{\text {int }}$ to generate the perturbation series. The following time ordered products enter our analysis:

$$
\begin{aligned}
& \left\langle T\left(\Theta^{ \pm}(x) \Theta^{ \pm}(y)\right)\right\rangle_{0}=\exp \left(-x D_{F}(x-y)\right)-1, \\
& \left\langle T\left(\Theta^{ \pm}(x) \Theta^{\mp}(y)\right)\right\rangle_{0}=\exp \left(+x D_{F}(x-y)\right)-1,
\end{aligned}
$$

where the righthand sides are obtained formally by Dyson-Wick reduction. $D_{F}(x-y)$ is the free propagator of a scalar particle. Because of the freedom of the generalised Stückelberg formalism, we can specialise to massless scalar particles.

We introduce the notation:

$$
\mathscr{D}^{ \pm}(x)=\exp \left( \pm \chi D_{F}(x)\right)-1
$$

It is not difficult to show that an arbitrary time ordered product $\left\langle T\left(\Theta^{ \pm}\left(x^{\prime}\right) \ldots \Theta^{\mp}\left(x_{n}\right) \ldots\right)\right\rangle_{0}$ involving any number of fields can be represented in terms of products of $\mathscr{D}(x)^{ \pm}$in (20). However, the expression (20) for $\mathscr{D}(x)^{ \pm}$is purely formal and it is necessary to obtain a more precise definition as a generalised function. One may then proceed 
to the analysis of more complicated matrix elements. Our method will consist first in introducing generalised Feynman amplitudes [1]. These are generalised functions, depending on parameters $\lambda$, defined on a fundamental space of test functions consistent with strict localizability. The physical amplitudes will then be obtained through analytic regularisation [10]. The next section is devoted first to defining the fundamental space and elucidating some of its essential properties. Subsequently the analytic method of obtaining a definition of $\mathscr{D}^{ \pm}(x)$ as a generalised function is studied.

\section{Section III. Analytic Regularisation}

\section{A. Test Function Spaces}

In order to motivate our choice of test function spaces let us consider the spectral function of the Green's function (20). On using the phase space representation of $N$ massless particles we get:

$$
\varrho_{ \pm}\left(p^{2}\right)=16 \pi^{3} \sum_{N=0}^{\infty} \frac{(\mp)^{N}\left(\frac{x p^{2}}{4 \pi^{2}}\right)^{N}}{\Gamma(N+3) \Gamma(N+2) \Gamma(N+1)} \pm \pi \varkappa \delta\left(p^{2}\right) .
$$

$\varrho_{ \pm}\left(p^{2}\right)$ is not a tempered distribution. However, it may be defined as a generalised function on certain test function spaces of type $S$ in which the condition of strict localizability may be satisfied. This condition implies that there should exist non zero test functions of compact support in this space. Constantinescu [11] has shown that spaces of type $S$ may be used, as an alternative to the Jaffe space [8] to formulate strictly local field theories and these spaces are sufficient for our purpose ${ }^{2}$. In particular we will use the test function space $S_{\beta}[9]$. However, the methods and proofs of this paper are also valid for Jaffe's space and the reader may interpret freely the space $\mathscr{C}$ (subsequently defined) as such. For convenience we state some relevant definitions.

We recall that the space $S_{\beta}\left(R^{1}\right)$, where $\beta$ is a non-negative number, consists of all test functions $\tilde{\varphi}(p)$ which are infinitely differentiable and satisfy the inequalities

$$
\left|p^{k} D^{(q)} \tilde{\varphi}(p)\right| \leqq C_{q} A^{k} k^{k \beta}
$$

where the constants $A$ and $C$ depend on $\tilde{\varphi}$.

An important restriction on the growths of the functions $\tilde{\varphi}(p)$ and its derivatives follows [9] from the inequality (22),

$$
\left|D^{(q)} \tilde{\varphi}(p)\right| \leqq C_{q}^{\prime} \exp \left(-b|p|^{1 / \beta}\right)
$$

\footnotetext{
${ }^{2}$ I am indebted to Professor J. G. Taylor for a helpful discussion.
} 
where

$$
b=\beta / e A^{1 / \beta} .
$$

Its extension to $\tilde{\varphi}(p), p \varepsilon R^{n}$, is:

$$
\left|D^{(q)} \tilde{\varphi}(p)\right| \leqq C_{q^{\prime}} \exp \left[-b\left(\left|p_{1}\right|^{1 / \beta}+\cdots+\left|p_{n}\right|^{1 / \beta}\right)\right] .
$$

The spaces $S_{\beta}$ are reciprocal in the sense that under Fourier transforms they pass into each other. We have [9]

$$
\tilde{S}_{\beta} \equiv F\left[S_{\beta}\right]=S^{\beta}
$$

and the Fourier operator is continuous in the topology of this space. The configuration space test functions in $S^{\beta}$ satisfy the conditions

$$
\left|x^{k} D^{(q)} \varphi(x)\right| \leqq C_{q} B^{q} q^{q \beta}
$$

where the constants $B, C$ depend on $\varphi$.

The topology of the space $S^{\beta}$ is studied in Ref. [9] where it is shown that it is a union of complete countably normed spaced with compatible norms.

The condition of strict localizability i.e. the condition that there exist non-zero configuration space test functions of compact support, can be satisfied with a restriction on $\beta$. Indeed we have the

Theorem. The space $S^{\beta}\left(x \in R^{n}\right)$ has at least one non-zero test function of compact support provided $\beta>1$.

The proof of this theorem follows from the considerations of Gelfand and Shilov [9] and we shall not elaborate on it here.

Furthermore, on the basis of considerations analogous to that of Ref. [8], the intersection $S^{\beta} \cap \mathscr{D}(\beta>1)$ is dense in $\mathscr{D}$ where $\mathscr{D}$ is the space of all infinitely differentiable functions of compact support. In the following we introduce the notation $\mathscr{C}\left(\equiv S^{\beta}, \beta>1\right)$ for the class of configuration space test functions and $\tilde{\mathscr{C}}\left(\equiv S_{\beta}, \beta>1\right)$ for the class of momentum space test functions. $\mathscr{C}^{\prime}$ and $\tilde{\mathscr{C}}^{\prime}$ denote the corresponding dual spaces of generalised functions.

It remains for us to check that the spectral functions $\varrho_{ \pm}\left(p^{2}\right)$ exist as generalised functions in $\tilde{\mathscr{C}}^{\prime}$. Now the convergence of the series (21) in the (weak) topology of $\tilde{\mathscr{C}}^{\prime}$ is assured if we majorise using (24) and take $\beta<\frac{3}{2}$. Then $\varrho_{ \pm}\left(p^{2}\right) \& \tilde{\mathscr{C}}^{\prime}$ by virtue of completeness of $\tilde{\mathscr{C}}^{\prime}$.

\section{B. Analytic Approach to Regularisation}

We now turn to the problem of giving distribution theoretic meaning to the two point function (20) of Section II. We start with the formal expression

$$
\mathscr{D}^{ \pm}(x)=\exp \left( \pm \varkappa D_{F}(x)\right)-1
$$


where

$$
D_{F}(x)=\frac{1}{4 \pi^{2}}\left(x^{2}+i o\right)^{-1}
$$

is the propagator of a massless particle [7]. We use the metric $x^{2}=x^{2}-x_{0}^{2}$.

In order to give meaning to (27) we shall consider a class of functionals in $\mathscr{C}^{\prime}$ depending on a complex parameter $\lambda[10]$. Let us write

$$
D_{F}(\lambda, x)=\frac{1}{4 \pi^{2}}\left(x^{2}+i o\right)^{\lambda-1}
$$

and

$$
\mathscr{D}^{ \pm}(\lambda, x)=\exp \left[ \pm \varkappa D_{F}(\lambda, x)\right]-1
$$

where we hold

$$
0<\operatorname{Re} \lambda<1, \quad \operatorname{Im} \lambda>0
$$

$\left(x^{2}+i o\right)^{\lambda-1}$ are tempered distributions meromorphic in $\lambda[10]$ with poles at $\lambda=-1-k(k=0,1,2, \ldots)$.

Now the series of tempered distributions

$$
\sum_{N=1}^{\infty} \frac{( \pm 1)^{N}\left(\frac{x}{4 \pi^{2}}\right)^{N}}{\Gamma(N+1)}\left(x^{2}+i o\right)^{N(\lambda-1)}
$$

converges in $\mathscr{C}^{\prime}$. From the continuity of the Fourier operator it follows that the Fourier transform of (30) may be constructed by taking the Four transform of (32) term by term. We then obtain by using standard results [10]

$\tilde{\mathscr{D}}^{ \pm}(\lambda, p)=\mathscr{I}\left(\mathscr{D}^{ \pm}(\lambda, x)\right)$

$=(4 \pi)^{2} \sum_{N=1}^{\infty} \frac{( \pm 1)^{N}\left(\frac{x}{4 \pi^{2}}\right)^{N} 2^{2 \lambda N}}{\Gamma(N+1)} \cdot \frac{\Gamma(2-(1-\lambda) N)}{\Gamma((1-\lambda) N)}\left(p^{2}-i o\right)^{(1-\lambda) N-2}$

$\tilde{\mathscr{D}}^{ \pm}(\lambda, p)$ exists as a generalised function in $\tilde{\mathscr{C}}^{\prime}$.

In the following we consider the restricted domain

$$
\Lambda:\{\lambda \mid 0<\operatorname{Re} \lambda<1,1>\operatorname{Im} \lambda>a>0\} .
$$

In the region $\Lambda, \tilde{\mathscr{D}}^{ \pm}(\lambda, p)$ is analytic. However, the physical point $\lambda=0$ is a singular point due to the poles of the numerator $\Gamma$ functions in the series (33). The $N^{\text {th }}$ term has poles at $\lambda=1-\frac{k+2}{N}$, where $k$ is any non-negative integer. Due to the infinite set of terms in the series, all these poles accumulate at $\lambda=0$ to give a non-isolated singularity at that point. Thus the wellknown method $[1,10]$ of extracting a regular part 
as a distribution by taking the constant term in the Laurent expansion in $\lambda$, which is valid for meromorphic distributions, cannot be applied here. We shall develop a new procedure.

In order to extract a regular part we first write (33) in the form of an integral representation. We do this by summing the series by Watson's method. We get

$$
\begin{aligned}
\tilde{\mathscr{D}}^{ \pm}(\lambda, p)=-\frac{\pi^{3} 2^{4}}{2 i} \int_{\Gamma} d z \frac{\left(\begin{array}{c}
\operatorname{Cos} \pi z \\
1
\end{array}\right)\left(2^{2 \lambda} \frac{x}{4 \pi^{2}}\right)^{z}}{\operatorname{Sin} \pi z \operatorname{Sin} \pi(1-\lambda) z} & \cdot \frac{\left(p^{2}-i o\right)^{(1-\lambda) z-2}}{\Gamma((1-\lambda) z-1) \Gamma((1-\lambda) z) \Gamma(z+1)}
\end{aligned}
$$

with $\lambda \varepsilon \Lambda$ as in (34).

By smearing (35) with a test function from $\tilde{\mathscr{C}}$ we find that the integral is meaningful in the sense of generalised functions ${ }^{3}$. The integrand in (35) is continuous in $z(\lambda \varepsilon \Lambda), z \varepsilon \Gamma$. The contour $\Gamma(|\operatorname{Im} z|<a)$ runs counterclockwise and encloses the positive real axis as shown in Fig. 1.

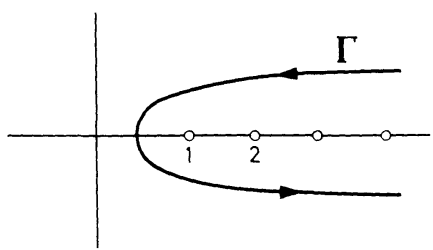

Fig. 1. The contour $\Gamma$ in the $Z$ plane

We now attempt to analytically continue $\tilde{\mathscr{D}}(\lambda, p)$ in $\lambda$ from the region $\Lambda$, where it is regular, to $\lambda=0$.

In the integrand of (35) there are two sets of poles in the $z$ plane. There are the fixed poles arising from $\sin \pi z$, and poles arising from $\operatorname{Sin} \pi(1-\lambda) z$, which move with $\lambda$. The latter poles are located at

$$
z=z_{n}=n(1-\lambda)^{-1}, \quad n=2,3, \ldots .
$$

With $\lambda \varepsilon \Lambda$ we have $0<\operatorname{Re} \lambda<1$ and $1>\operatorname{Im} \lambda>a>0$. Thus the poles at $z_{n}$ are in the upper quadrant of the right half $z$ plane exterior to the contour $\Gamma$. We now continue in $\lambda$ towards $\lambda=0$. The motion of the poles at $z_{n}(\lambda)$ is shown in Fig. 2.

It is evident that in the process of continuation a pinch singularity is generated at $\lambda=0$, since the contour gets trapped between the $z_{n}$ poles and the fixed poles at the positive integers and one cannot escape the trap by contour deformation. We now turn to extracting the singularity.

\footnotetext{
${ }^{3}$ See Appendix 2, p. 137 of Ref. [10]
} 


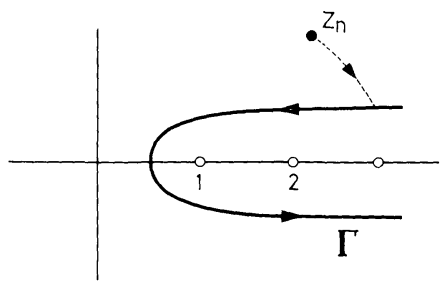

Fig. 2. Motion of poles at $Z_{n}(\hat{\lambda})$ in the $Z$ plane

We can rewrite (35) by deforming the contour $\Gamma$ so that the $z_{n}$ poles are now enclosed by it and using Cauchy's theorem to compute the discontinuity.

We obtain

where

$$
\tilde{\mathscr{D}}^{ \pm}(\lambda, p)=\tilde{\mathscr{D}}_{R}^{ \pm}(\lambda, p)+\Delta D^{ \pm}(\lambda, p)
$$

$$
\begin{aligned}
& \tilde{\mathscr{D}}_{R}^{ \pm}(\lambda, p)=-\frac{\pi^{3} 2^{4}}{2 i} \int_{\bar{I}} d z \frac{\left(\begin{array}{c}
\operatorname{Cos} \pi z \\
1
\end{array}\right)\left(2^{2 \lambda} \frac{\varkappa}{4 \pi^{2}}\right)^{z}}{\operatorname{Sin} \pi z \operatorname{Sin} \pi(1-\lambda) z} \\
& \frac{\left(p^{2}-i o\right)^{(1-\lambda) z-2}}{\Gamma((1-\lambda) z-1) \Gamma((1-\lambda) z) \Gamma(z+1)}
\end{aligned}
$$

and the discontinuity is given by

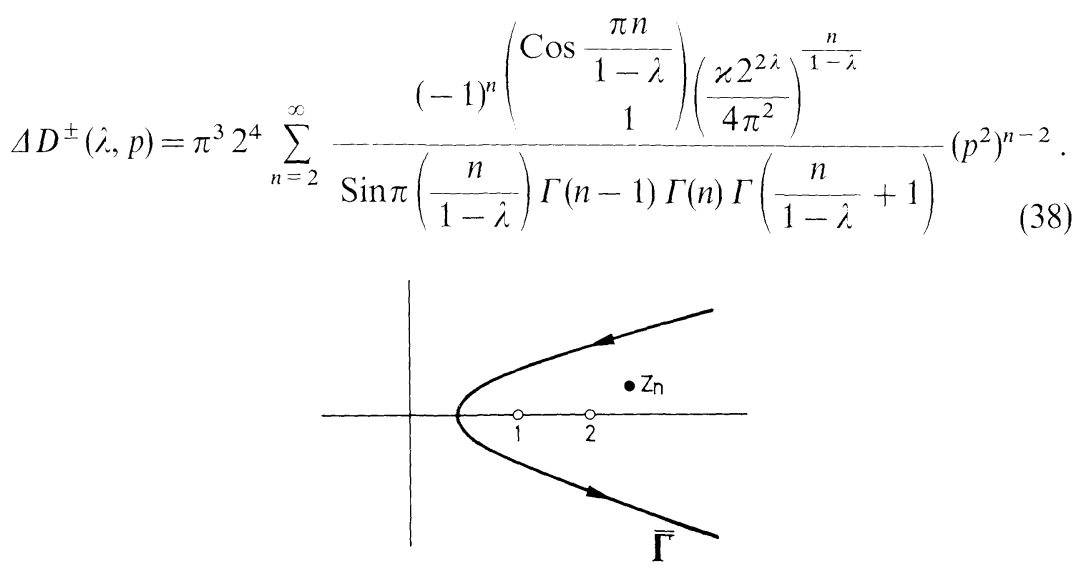

Fig. 3. The contour $\bar{\Gamma}$ in the $Z$-plane

We hold $\lambda \varepsilon \bar{\Lambda}$ where $\bar{\Lambda}$ is the region $o<\operatorname{Re} \lambda<1$ and $a>\operatorname{Im} \lambda>0$ and the contour $\bar{\Gamma}$ in the definition (37) of $\tilde{\mathscr{P}} \pm(\lambda, p)$ is shown in Fig. 3.

$\tilde{\mathscr{D}}_{(R)}^{ \pm}(\lambda, \mathrm{p})$ is analytic in $\lambda$ at $\lambda=0$ and may be continued to that point. On the other hand $\Delta D^{ \pm}(\lambda, p)$ contains a non-isolated singularity at $\lambda=0$. 
We now define the generalised function $\tilde{\mathscr{D}}^{ \pm}(\lambda, p)$ at $\lambda=0$ by discarding the non-meromorphic discontinuity $\Delta D^{ \pm}(\lambda, p)$ :

$$
\tilde{\mathscr{D}}^{ \pm}(p)=\underset{\lambda \rightarrow 0}{\operatorname{Anal}} \operatorname{Cont} \tilde{\mathscr{D}}_{(R)}^{ \pm}(\lambda, p) .
$$

We have $\tilde{\mathscr{D}}^{ \pm}(p) \varepsilon \tilde{\mathscr{C}}^{\prime}$ since $\mathscr{D}_{(R)}^{ \pm}(\lambda, p) \varepsilon \tilde{\mathscr{C}}^{\prime}$ and is analytic at $\lambda=0$. Finally, evaluating (39) by using Cauchy's theorem we get

$$
\begin{aligned}
& \tilde{\mathscr{D}}^{ \pm}(p)= \pm \frac{x}{p^{2}-i o}-\left(\frac{4 \pi}{p^{2}}\right)^{2} \sum_{n=2}^{\infty} \frac{(\mp 1)^{n}\left(\frac{x p^{2}}{(4 \pi)^{2}}\right)^{n}}{\Gamma(n-1) \Gamma(n) \Gamma(n+1)} \\
& \text { where } \quad \cdot\left\{\log \left(\frac{x\left(p^{2}-i o\right)}{4 \pi^{2}}\right)-\psi(n-1)-\psi(n)-\psi(n+1)\right\} .
\end{aligned}
$$

$$
x=\left(\frac{2 g}{m_{w}}\right)^{2} .
$$

The imaginary part of $(40)$ is $\varrho^{ \pm}\left(p^{2}\right)$ which was discussed as a generalised function in $\tilde{\mathscr{C}}^{\prime}$ in subsection $A$. The configuration space propagator $\mathscr{D}^{ \pm}(x)$ is now defined as the Fourier transform of $\tilde{D}^{ \pm}(p)$ and is a generalised function in $\mathscr{C}^{\prime}$.

The result (40) had been obtained earlier by heuristic methods by Volkov [2] and Okubo [3]. A special feature is the emergence of a logarithmic dependence on the coupling constant " $g$ " and consequent lack of analyticity in " $g$ ". Now the regularisation (40) has been achieved by discarding the non-isolated singularity at $\lambda=0$ which, in configuration space, ic concentrated at the light cone vertex since (38) represents an entire function of order less than one half. In configuration space it amounts to a freedom in adding to $\mathscr{D}^{ \pm}(x)$ arbitrary functionals of point support in $\mathscr{C}^{\prime}$ to obtain different choices for the propagator. Such functionals have the form $f(\square) \delta(x)$ where $f(s)$ is an entire function of $s$ of type zero and order less than half. We note that precisely the same definition of $\mathscr{D}^{ \pm}(x)$ as a distribution has been independently obtained through an alternative route by Lehmann and Pohlmeyer [4]. From their work it is known in addition that $\mathscr{D}^{ \pm}(x)$ does not contain any distribution concentrated at the vertex of the light cone ${ }^{4}$. This choice for the propagator is the smoothest possible one (the minimum singularity hypothesis [4]) and the addition of further functionals of point support would imply the addition of counter terms to $\mathscr{L}_{] p[}[7]$. In the following we shall work with the smooth propagator $\mathscr{D}^{ \pm}(x)$.

\footnotetext{
${ }^{4}$ An unambiguous characterisation is given in Ref. [4].
} 


\section{Section IV. Higher Orders; Renormalisation of Loop Diagrams}

Having obtained the exponential interaction propagator as a distribution $\mathscr{D}^{ \pm}(x)$ our next task is to examine its insertion in loop diagrams. The renormalisation problem is that of giving a rule for multiplying the distributions in $\mathscr{C}^{\prime}$ so as to obtain further distributions in $\mathscr{C}^{\prime}$ which are fixed up to functionals concentrated at the origin of $R^{n}$. In this section we consider the simplest case: that in which a single super-propagator $\mathscr{D}^{ \pm}(x)$ is involved together with any other propagator (e.g. a spinor line). From the present discussion the outline of the solution for the general case (in which any number of $\mathscr{D}^{ \pm}(x)$ are involved) will emerge, although we will postpone the systematic discussion of the general case to another occasion.

We consider specifically the fermion self-energy part which arises on two iterations of $\mathscr{L}_{\text {int }} . \Sigma_{\theta}^{(2)}(p)$ is the real object of concern and corresponds formally to the graphs of Fig. 4.

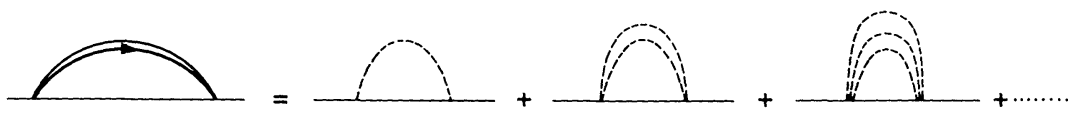

Fig. 4. The self energy part $\Sigma_{\theta}$

In configuration space we have to consider the product $\mathscr{D}^{ \pm}(x) S_{F}(x)$ where $\mathscr{D}^{ \pm}(x)$ is the super propagator defined in the previous section as the Fourier transform of $\tilde{D}^{ \pm}(p)$. Although $S_{F}(x)$ and, now, $\mathscr{D}^{ \pm}(x)$ are well defined as distributions, their product is yet undefined and our task is to give meaning to it.

To this end we define as usual [1] generalised propagators:

where

$$
\Delta_{F}(x, \lambda)=\mathscr{I}(\tilde{\Delta}(p, \lambda))
$$

$$
\tilde{\Delta}(p, \lambda)=\left(p^{2}+m^{2}-i o\right)^{-(\lambda+1)}
$$

and ignore (for our purposes) the inessential complication of the spin of the fermion. (The following arguments can be easily generalised.)

We also define an analytic extension of $\mathscr{D}^{ \pm}(x)$ :

$$
\begin{gathered}
\mathscr{D}^{ \pm}(x, \lambda)=\mathscr{I}\left(\tilde{\mathscr{D}}_{ \pm}(p, \lambda)\right), \\
\tilde{\mathscr{D}}_{ \pm}(p, \lambda)=-\frac{\pi^{3} 2^{4}}{2 i} \int_{\Gamma} d z \frac{\left(\begin{array}{c}
\operatorname{Cos} \pi z \\
1
\end{array}\right)\left(2^{2 \lambda} \frac{x}{4 \pi^{2}}\right)^{z}}{\operatorname{Sin}^{2} \pi z \Gamma(z+1) \Gamma(z)} \cdot \frac{\left(p^{2}+\mu^{2}-i o\right)^{(1-\lambda) z-2}}{\Gamma((1-\lambda) z-1)} .
\end{gathered}
$$

Note that the propagator in (45) is not to be confused with (34). In the above we shall hold $\operatorname{Im} \lambda>a>0$. Then $\tilde{\mathscr{D}}^{ \pm}(p, \lambda)$ is analytic in $\lambda$. We have 
introduced the real parameter $\mu$ to control "infra-red" divergences. We will show that the limit $\mu \rightarrow 0$ can be smoothly taken in the analytically renormalised amplitude.

In configuration space we have:

$$
\begin{aligned}
\mathscr{D}^{ \pm}(x, \lambda)=\int_{\Gamma} d z \frac{\left(\begin{array}{c}
\operatorname{Cos} \pi z \\
1
\end{array}\right)\left(2^{\lambda+1} \frac{x}{4 \pi^{2}}\right)^{z}}{\operatorname{Sin}^{2} \pi z \Gamma(z+1) \Gamma((1-\lambda) z-1) \Gamma(z)} \\
\cdot \frac{\left(\mu^{2}\right)^{(1-\lambda) z} K_{(1-\lambda) z}\left(\mu\left(x^{2}+i o\right)^{\frac{1}{2}}\right)}{\Gamma(2-(1-\lambda) z)\left(x^{2}+i o\right)^{\frac{1}{2}(1-\lambda) z}} .
\end{aligned}
$$

In the following " $i o$ " should be understood as " $i \varepsilon$ " and the limit $\varepsilon \rightarrow 0$ is taken in the end in the sense of distributions. With $3>\operatorname{Re} \lambda>1$, $1>\operatorname{Im} \lambda>a>0, \tilde{\Delta}_{F}(\lambda, p)$ and $\tilde{\mathscr{D}}^{ \pm}(\lambda, p)$ are $L^{1}$ functions. The $L^{1}$ nature of $\tilde{\mathscr{D}}^{ \pm}(\lambda, p)$ follows from the convergence of its series representation in the $L^{1}$ norm. Thus the product $\Delta_{F}\left(\lambda_{2}, x\right) \times \mathscr{D}^{ \pm}\left(\lambda_{1}, x\right)\left(\sim \Sigma_{ \pm}(x)\right)$ is well defined and can be obtained as $\mathscr{I}\left(\Sigma^{ \pm}(p, \lambda)\right)$ where $\Sigma^{ \pm}(p, \lambda)$ is the convolution of $\tilde{\Delta}_{F}$ with $\tilde{\mathscr{D}}^{ \pm}$. On using the continuity of the convolution operation we get

$$
\begin{aligned}
& \Sigma^{ \pm}(\lambda, p)=\int_{\Gamma} d z \frac{\left(\begin{array}{c}
\operatorname{Cos} \pi z \\
1
\end{array}\right)\left(2^{2 \lambda} \frac{x}{4 \pi^{2}}\right)^{z}}{\operatorname{Sin}^{2} \pi z \Gamma(z+1) \Gamma(z) \Gamma((1-\lambda) z-1)} \\
& \cdot \int d^{4} k\left(m^{2}+(p-k)^{2}-i \varepsilon\right)^{-1}\left(k^{2}+\mu^{2}-i \varepsilon\right)^{(1-\lambda) z-2} .
\end{aligned}
$$

In (47) we have set $\lambda_{1}=\lambda$ and $\lambda_{2}=0$ which does not affect the convergence of the convolution integral (which we call $J(\lambda, z ; p)$ ) and simplifies the analysis. From the standard analysis [1] it is known that $J(\lambda, z ; p)$ is meromorphic in the combination $(1-\lambda) z$ so that the search for the $\lambda$ singularity may be conducted as in the previous section. More precisely, we get, after Feynman parametrisation [7] and integration in the variable corresponding to the sum of the parameters,

$$
\begin{aligned}
J(\lambda, z ; p)=i \pi^{3}\left(m^{2}\right)^{(1-\lambda) z-1} & \frac{\Gamma(1-(1-\lambda) z)}{\Gamma(2-(1-\lambda) z)} \\
& \cdot \int_{0}^{1} d \xi \xi^{1-(1-\lambda) z}\left[(1-\xi)\left(1+\xi \frac{p^{2}-i \varepsilon}{m^{2}}\right)+\mu^{2} \xi\right]^{(1-\lambda) z-1}
\end{aligned}
$$

the meromorphy of $J(\lambda, z ; p)$ in $(1-\lambda) z$ follows on integration by parts. We can now write:

$$
\Sigma^{ \pm}(\lambda, p)=\int_{\Gamma} d z \frac{\left(\begin{array}{c}
\operatorname{Cos} \pi z \\
1
\end{array}\right)\left(2^{2 \lambda} \frac{x}{4 \pi^{2}}\right)^{z}}{\operatorname{Sin}^{2} \pi z \Gamma(z+1) \Gamma(z) \Gamma((1-\lambda) z-1) \operatorname{Sin} \pi(1-\lambda) z} \mathscr{J}(\lambda, z ; p) .
$$


Where

$$
\begin{aligned}
& \mathscr{J}(\lambda, z ; p)=i \pi^{3}\left(m^{2}\right)^{(1-\lambda) z-1} \frac{\Gamma(1-(1-\lambda) z)}{\Gamma(2-(1-\lambda) z)} \\
& \cdot \operatorname{Sin} \pi(1-\lambda) z \int_{0}^{1} d \xi \xi^{-(1-\lambda) z+1}\left[(1-\xi)\left(1+\xi \frac{p^{2}-i \varepsilon}{m^{2}}\right)+\mu^{2} \xi\right]^{(1-\lambda) z-1}
\end{aligned}
$$

Clearly $\mathscr{J}(\lambda, z ; p)$ is entire in $\lambda$. Thus it can be analytically continued to the region $\operatorname{Re} \lambda<1$. Returning to (49) we continue in $\lambda$ to the region $\operatorname{Re} \hat{\lambda}<1,1>\operatorname{Im} \lambda>a>0$. In doing so the moving $Z_{n}(\lambda)$ poles, arising from $\operatorname{Sin} \pi(1-\lambda) z$ (see Section III) migrate from the upper left to the upper right quadrant in the $z$ plane, but, since $\operatorname{Im} \lambda>a$, they can reside in the exterior of the contour $\Gamma$. With $\operatorname{Re} \lambda<1, \operatorname{Im} \lambda>a>0$ the analytic continuation of $(50)$ is given by:

$$
\begin{aligned}
& \mathscr{J}(\lambda, z ; p)=i \pi^{3}\left(m^{2}\right)^{(1-\lambda) z-1} \frac{\Gamma(1-(1-\lambda) z)}{\Gamma(2-(1-\lambda) z)} \\
& \quad \cdot i e^{i \pi(1-\lambda) z} \int_{1}^{0+} d \xi \xi^{1-(1-\lambda) z}\left[(1-\xi)\left(1+\xi \frac{p^{2}-i \varepsilon}{m^{2}}\right)+\mu^{2} \xi\right]^{(1-\lambda) z-1}
\end{aligned}
$$

where the contour begins and ends at 1 encircling the origin once counterclockwise, and hold $\varepsilon>0$. The representation (51) can be obtained from (50) by first restricting to the region $0<\operatorname{Re}(1-\lambda) z<2$. In that region writing the integral in (50) as:

$\left(\mu^{2}\right)^{(1-\lambda) z-1} \int_{0}^{1} d \xi \xi^{1-(1-\lambda) z}\left[1+\frac{1-\mu^{2}}{\mu^{2}}(1-\xi)\left(1+\xi \frac{p^{2}-i \varepsilon}{m^{2}\left(1-\mu^{2}\right)}\right)\right]^{(1-\lambda) z-1}$

then expanding the integrand in a binomial series, next employing a standard contour representation for hypergeometric functions [12] and summing back we derive (51). Then the representation (51) continues to hold in the region of interest $\operatorname{Re} \lambda<1, \operatorname{Re} z>0$ (arbitrary) since both sides are analytic.

At this stage $(\operatorname{Re} \lambda<1,1>\operatorname{Im} \lambda>a>0)$ we note that the boundary value $\varepsilon \rightarrow 0$ of (49) is a distribution in $\tilde{\mathscr{C}}^{\prime}$. This follows [1] by smearing (51) (a tempered distribution), with a test function from $\tilde{\mathscr{C}}$ and examining the convergence of the $Z$ integration in (49). (The conditions of the Lebesgue bounded convergence theorem are satisfied. We use it in two stages (i) to interchange $\xi, z$ integration (the $z$-integral corresponds to a series) (ii) to prove the existence of the $\varepsilon \rightarrow 0$ limit [1].) We also note that the limit $\mu^{2} \rightarrow 0$ can now be taken smoothly in the sense of distributions as asserted earlier. We can now extract the $\lambda$ singularity using the method of Section III. As we continue in $\lambda$ to $\lambda=0$ the moving $Z_{n}(\lambda)$ singularities trap the contour $\Gamma$ against the fixed poles at $Z=n$ leading finally to a 
pinch. We deform the contour $\Gamma \rightarrow \bar{\Gamma}$, as before, so as to enclose the $Z_{n}(\lambda)$ poles and compute the discontinuity. Then:

$$
\Sigma^{ \pm}(\lambda, p)=\Sigma_{R}^{ \pm}(\lambda, p)+\Delta \Sigma^{ \pm}(\lambda, p),
$$

$\Sigma_{R}$ is $\Sigma$ with the contour $\Gamma$ replaced by $\bar{\Gamma}$ and $\Delta \Sigma$ is the discontinuity. $\Sigma_{R}$ is analytic at $\lambda=0$ whereas $\Delta \Sigma$ contains a non-isolated singularity at $\lambda=0$. We can now define $\Sigma^{ \pm}$at $\lambda=0$ as a distribution in $\tilde{\mathscr{C}}^{\prime}$ :

$$
\Sigma^{ \pm}(p)=\text { Anal. Cont } \Sigma_{i}^{ \pm}(\lambda, p) .
$$

We then get:

where

$$
\Sigma^{ \pm}(p)=\int_{\bar{\Gamma}} d z \frac{\left(\begin{array}{c}
\operatorname{Cos} \pi z \\
1
\end{array}\right)\left(\frac{\varkappa}{4 \pi^{2}}\right)^{z}}{\operatorname{Sin}^{3} \pi z \Gamma(z+1) \Gamma(z) \Gamma(z-1)} \mathscr{J}(z ; p)
$$

$$
\begin{aligned}
\mathscr{J}(z ; p)=i \pi^{3}\left(m^{2}\right)^{z-1} & \Gamma(1-z) \Gamma(2-z)^{-1} \\
& \cdot i e^{i \pi z} \int_{1}^{0+} d \xi \xi^{1-z}(1-\xi)^{z-1}\left(1+\xi \frac{p^{2}-i o}{m^{2}}\right)^{z-1} .
\end{aligned}
$$

The expression (56) can also be identified with a hypergeometric function $[12]$ :

$$
\mathscr{J}(z ; p)=i \pi^{3}\left(m^{2}\right)^{z-1} F\left(1-z, 2-z ; 2 ;-\frac{p^{2}-i o}{m^{2}}\right) .
$$

From (57) and (55) more detailed expressions can be obtained by using series expansions of (57) and employing the residue calculus. These expressions which display a non-analytic dependence on $\%$ agree precisely with those obtained earlier by Volkov [2] and are now seen to have a precise distribution theoretic significance.

We now discuss two further points connected with finite renormalisation ambiguity and smoothness.

Since we have extended a continuous linear functional defined on a subspace of $C^{\infty}$ test functions which vanish, together with all its derivatives, at the origin to $\mathscr{C}^{\prime}$ in principle we are free to add a distribution concentrated at the origin. This can be seen explicitly from our renormalisation procedure. In computing the discontinuity $\Delta \Sigma^{ \pm}$(which we subsequently subtract off) we have to evaluate $\Sigma^{ \pm}(\hat{\lambda}, p)$ using (51) and taking only the contributions from the moving poles at $z=z_{n}=n(1-\lambda)^{-1}$. The residues of the poles are polynomials in $p^{2}$ (since (51) is now trivially evaluated using residue theory). The polynomials are easily recognisable (degenerate case of a hypergeometric function), and the sum is an entire function of $p^{2}$ of order less than half. In configuration space the subtractional ambiguity is a distribution concentrated at the vertex of the light cone. This also shows that unitarity remains unaffected as one may verify tediously. 
We now turn to the question whether in spite of the above "in principle" ambiguity there is some sense in which the renormalised amptitude is unique. For this purpose we pass smoothly to the $m=0$ limit in $\Sigma^{ \pm}(p)$. In that limit we recover essentially the super propagator $\tilde{\mathscr{D}}^{ \pm}(p)$ (except for a trivial combinatorial difference which is expected). Then, [2], $\Sigma^{-}\left(\operatorname{Re} \Sigma^{+}\right)$is strongly decreasing as $p^{2} \rightarrow \pm \infty$ (a $G$-function [12]). Then smearing e.g. $\Sigma^{-}(x)$ with a test function $\varphi\left(x^{0}\right)$ we show that it is $C^{\infty}$ in the remaining variables [4]. Since we expect the leading short distance singularity to be independent of the external mass " $m$ " $\Sigma^{ \pm}$ is maximally smooth and the addition of any further distribution concentrated at $x=0$ (equivalently a counterterm) would destroy this property.

The method we have given for loop integrals involving a single super propagator can, in principle, be extended to the case when any number of $\mathscr{D}^{ \pm}(x, \lambda)$ are involved. This will happen in arbitrary orders of perturbation theory in the exponential interaction.

The formal product $\prod_{l} \mathscr{D}_{(l)}^{ \pm}\left(x_{a}-x_{b}\right)$ where $l$ indexes each superpropagator $)$ is to be replaced by $\prod_{l} \mathscr{D}_{(l)}^{ \pm}\left(x_{a}-x_{b} ; \lambda_{l}\right)$ with $\operatorname{Re} \lambda_{l}>1$ and this is well defined since each $\tilde{\mathscr{D}}_{(l)}\left(p, \lambda_{l}\right)$ is $L^{1}$; the convolution now leads to a well defined momentum space representation as a distribution in $\tilde{\mathscr{C}}^{\prime}$.

The analysis of singularities in the components $\lambda_{i}$ of the $\lambda$ has to be repeated. Consider the generalisation of Eq. (47) to this case. Then it is known [1] that the momentum space integral is a meromorphic function of the combinations $\left(1-\lambda_{i}\right) Z_{i}(i=1 \ldots n)$. We would expect a similar pinch analysis to lead to a regular part as a distribution in $\tilde{\mathscr{C}}^{\prime}$. However, this problem needs further precise discussion and we shall return to it elsewhere.

\section{Section V. Concluding Remarks}

Given a classical Lagrangian it is well known that the quantum Lagrangian in perturbation theory is fixed only after completing the definition of $T$-products for coinciding arguments [7]. The axioms of local field theory require that the defined $T$-products be localisable distributions. The choice is arbitrary to the extent that one is free to add to the definitions distributions concentrated at the origin of $R^{n}\left(X_{1}=X_{2}=\cdots=X_{n}=0\right)$ and these have interpretations as counterterms [7]. We have shown, through the simple cases, how the analytic method might be exploited to complete the definition of $T$-products as unitary localisable distributions for the present theory.

The question naturally arises as to whether the considerable finite renormalisation arbitrariness can be further restricted. For this we find 
most appealing the introduction of the notion of "minimum singularity" by Lehmann and Pohlmeyer [4] in order to define the least singular (perturbative) quantum field theory corresponding to the given classical interaction. This amounts to selecting the smoothest regularised amplitudes (in the sense of absence of distributions concentrated at the light cone vertex). This hypothesis would eliminate finite renormalisation counter terms associated with the exponential interaction. It is known that the Froissart bound holds [13] for strictly localisable field theories and one may hope that in some way it is the least singular perturbative amplitudes which sum up correctly. Thus, in the context of analytic renormalisation, the main problem is to make more systematic the discussion of higher orders especially in relation to the property of smoothness. For the simple cases the analytically renormalised amplitudes enjoy this property. Given the lack of an appealing local renormalisable theory of weak interactions these questions would appear to warrant further discussion.

Acknowledgements. I am deeply indebted to Professor H. Lehmann for many stimulating conversations and to Professor M. Lévy and D. Bessis for hospitality at the Institut d'Etudes Scientifiques de Cargese. I thank Dr. J. C. Taylor for helpful conversations, Professor Sir Rudolf Peierls for his hospitality at Oxford and the Science Research Council for support.

\title{
References
}

1. Speer, E.: Generalised Feynman amplitudes, Annals of Mathematics Studies, No. 62, Princeton University Press.

2. Volkov, M.: Ann. Phys. 49, 202 (1968); Commun. math. Phys. 7, 289 (1968).

3. Okubo, S.: Prog. Theor. Phys. 11, 80 (1954).

4. Lehmann, H., Pohlmeyer, K.: DESY preprint, June 1970.

5. Constantinescu, F.: Munich preprint, July 1970.

6. Lee, T. D.: Nuovo Cimento 59 A, 579 (1969).

7. Bogoliubov, N., Shirkov, D.: Theory of quantized fields. New York: Interscience Publishers 1959.

8. Jaffe, A.: Phys. Rev. 158, 1454 (1967).

9. Gelfand, I. M., Shilov, G. E.: Generalised functions, Vol. 2, Chapter IV. New York: Academic Press 1968.

10. -- - Generalised functions, Vol. 1, New York: Academic Press 1964.

11. Constantinescu, F.: Munich preprints (1969).

12. Higher transcendentral functions, Ed. Erdelyi, Vol. I. New York: McGraw-Hill 1953.

13. Epstein, H., Glaser, A., Martin, A.: Commun. math. Phys. 13, 157 (1969).

\author{
P. K. Mitter \\ University of Oxford \\ Department of Theoretical Physics \\ 12 Parks Road \\ Oxford OX 1 3PQ/England
}

